\title{
Outcomes of transperineal and transrectal ultrasound-guided prostate biopsy
}

\author{
KL Lo, KL Chui, CH Leung, SF Ma, Kevin Lim, Timothy Ng, Julius Wong, Joseph KM Li, SK Mak, CF Ng *
}

\section{A B S T R A C T}

Objective: To compare the clinical outcomes and pathological findings of transperineal ultrasoundguided prostate biopsy (TPUSPB) and transrectal ultrasound-guided prostate biopsy (TRUSPB) in a secondary referral hospital.

Methods: This was a retrospective study of 100 TPUSPBs and 100 TRUSPBs performed in our centre. Pre-biopsy patient parameters (eg, patient age, clinical staging, serum prostate-specific antigen [PSA] level, prostate size, and PSA density), as well as pathological results and 30-day complication and readmission rates, were retrieved from the patients' medical records and compared between the two groups.

Results: One hundred TPUSPBs performed from January 2018 to May 2018 and 100 TRUSPBs performed from January 2016 to April 2016 were included for analysis. Mean age did not significantly differ between the groups. The TPUSPB group had a higher mean PSA level, smaller prostate size, and higher PSA density, compared with the TRUSPB group. The overall prostate cancer detection rate was similar between the TPUSPB and TRUSPB groups ( $35 \%$ vs $25 \%, \mathrm{P}=0.123$ ). There were no significant differences between the groups in prostate cancer
PSA density and clinical staging. With respect to complications, no patients developed fever in the TPUSPB group, while $4 \%$ of patients in the TRUSPB group had fever and required at least 1-week admission for intravenous antibiotic administration.

Conclusion: For prostate biopsy, TPUSPB is safer, with no infection complications, and has similar prostate cancer detection rate compared with TRUSPB.

\section{Hong Kong Med J 2019;25:209-15 \\ https://doi.org/10.12809/hkmj187599}

\author{
${ }^{1} \mathrm{KL}$ LO, MB, ChB, FHKAM (Surgery) \\ ${ }^{1} \mathrm{KL}$ Chui, MB, BS, FHKAM (Surgery) \\ ${ }^{2} \mathrm{CH}$ Leung, MSc \\ SF Ma, MB, ChB \\ ${ }^{1} \mathrm{~K}$ Lim, MB, ChB \\ ${ }^{1} \mathrm{~T} \mathrm{Ng}, \mathrm{MB}, \mathrm{ChB}$ \\ $1 \mathrm{~J}$ Wong, $\mathrm{MB}, \mathrm{ChB}$ \\ ${ }^{1} \mathrm{JKM}$ Li, MB, ChB, FHKAM (Surgery) \\ ${ }^{1}$ SK Mak, MB, BS, FHKAM (Surgery) \\ ${ }_{1,2} \mathrm{CF} \mathrm{Ng}{ }^{*}, \mathrm{MB}, \mathrm{ChB}$, FHKAM (Surgery) \\ Division of Urology, North District Hospital, New Territories East Cluster \\ Urology Unit, Prince of Wales Hospital, Shatin, Hong Kong \\ 2 SH Ho Urology Centre, Department of Surgery, The Chinese University \\ of Hong Kong, Shatin, Hong Kong
}

detection rates after stratification according to * Corresponding author: ngcf@surgery.cuhk.edu.hk

New knowledge added by this study

- There were no sepsis complications associated with the use of transperineal prostate biopsy (TPUSPB), which avoids penetration of the rectal mucosa and possible transfer of intestinal flora to the blood stream during the procedure.

- In terms of prostate cancer detection, TPUSPB was comparable to transrectal prostate biopsy (TRUSPB). Moreover, TPUSPB may have an advantage over TRUSPB in patients with previous negative biopsy findings, as it does not neglect prostate cancer in the anterior fibromuscular stroma.

Implications for clinical practice or policy

- TPUSPB is suitable for use as a routine, 1-day out-patient procedure, which may be helpful for patients who must travel a considerable distance to reach the hospital.

- TPUSPB may be more suitable for patients who cannot undergo general anaesthesia or monitored anaesthesia care.

- TPUSPB might be a good alternative to TRUSPB, particularly for patients with increased risk of sepsis.

\section{Introduction}

According to the Hong Kong Cancer Registry, prostate cancer is the third most common cancer in men. As in other cancers, biopsy is needed for histological confirmation of the diagnosis of prostate cancer before treatment is initiated. With the increasing age of the population, the incidence rate of this cancer is expected to increase; the frequency of prostate biopsy will therefore also increase. Hodge et $\mathrm{al}^{2}$ introduced the systematic sextant biopsy protocol under transrectal ultrasound guidance. Transrectal ultrasound-guided prostate biopsy (TRUSPB) has since become a widely accepted and routinely performed technique to detect prostate cancer. ${ }^{3}$ 


\section{經會陰和經直腸超聲前列腺活組織檢查的結果 羅家麟、崔家倫、梁志豪、馬肇暉、林諾賢、伍正傑、 黃浩輝、李啟旻、麥肇敬、吳志輝}

目的：比較經會陰超聲前列腺活組織檢查（TPUSPB）和經直腸超聲 前列腺活組織檢查（TRUSPB）在地區醫院的臨床和病理結果。

方法：我們以本中心進行的 100 個TPUSPB和 100 個TRUSPB作回顧性 研究。從兩組患者的病歷中檢索並比較活檢前的患者參數（如患者年 齡、臨床分期、血清前列腺特異性抗原〔PSA 水平、前列腺大小和 PSA密度), 以及病理結果和 30 天併發症和再入院率等資料。

結果：我們將2018年1月至2018年5月期間進行的100個TPUSPB與 2016 年1月至 2016 年 4 月期間進行的 100 個TRUSPB進行分析。兩組 間的平均年齡沒有顯著差異。與TRUSPB組相比, TPUSPB組的平 均PSA水平較高、前列腺較細和PSA密度較高。TPUSPB和TRUSPB 組的總前列腺癌檢出率相似（ $35 \%$ 比 $25 \%, \mathrm{P}=0.123$ ）。根據PSA密 度和臨床分期, 分層後前列腺癌檢出率組間無顯著差異。併發症方 面, TPUSPB組沒有患者出現發熱, TRUSPB組中有4\%的患者發燒, 並且需要至少 1 週的靜脈內抗生素治療。

結論：與TRUSPB相比, TPUSPB更安全和無感染併發症, 前列腺癌 檢出率也相若。 be performed without antibiotic prophylaxis, thus reducing the risk of generating antibiotic resistance. ${ }^{4}$ Based on these potential benefits, our centre introduced TPUSPB beginning in January 2018. Subsequently, we have completely replaced TRUSPB with TPUSPB. In this study, we aimed to compare the outcomes of our initial series of patients who underwent TPUSPB with those of our previous cohort of patients who underwent TRUSPB.

\section{Methods}

In this retrospective cohort study, we compared 100 patients who underwent TPUSPB with 100 patients who underwent TRUSPB in our centre. This study was approved by our institutional ethics committee. All 100 patients who underwent TPUSPB from January 2018 to May 2018 (TPUSPB group) were included; 100 patients who underwent TRUSPB from January 2016 to April 2016 were also included. The indications for biopsy for both groups were serum PSA $>4 \mathrm{ng} / \mathrm{dL}$, abnormal digital rectal examination, and surveillance biopsy for patients under active surveillance.

The following data were retrieved from hospital records and compared between the two groups: age, serum PSA level, prostate size, PSA density, prostate cancer detection rate, and complications (eg, admission due to acute retention of urine, rectal bleeding, haematuria, fever, and sepsis). We used the Third International Consensus Definitions for Sepsis and Septic Shock (Sepsis-3) as an acute change in total sequential organ failure assessment score $\geq 2$ points due to the infection ${ }^{11}$ : (1) respiratory rate $\geq 22 /$ min, (2) altered mental activity, and (3) systolic blood pressure $\leq 100 \mathrm{~mm} \mathrm{Hg}$.

For both groups, the indications for prostatic biopsies were serum PSA level $>4 \mathrm{ng} / \mathrm{dL}$, abnormal digital rectal examination, or follow-up biopsy for patients under active surveillance. All patients underwent pre-procedure blood tests and urine tests to ensure there was no bleeding tendency or positive urine culture. Patients using antiplatelet or anticoagulant treatment were required to discontinue drugs prior to undergoing biopsy. All patients used a sodium phosphate rectal enema in the morning of the procedure and took oral prophylactic antibiotics $(1 \mathrm{~g}$ amoxicillin-clavulanate and $500 \mathrm{mg}$ ciprofloxacin) 2 hours before the procedure. For TPUSPB, numbing cream (2.5\% lidocaine and $2.5 \%$ prilocaine) was applied over the perineal region 1 hour before the procedure and $1 \%$ lidocaine $(20 \mathrm{~mL})$ was injected into the perineum as local anaesthesia (LA) immediately prior to prostate biopsy, at a 45degree angle from the midline and approximately $15 \mathrm{~mm}$ above the anus on either side. Details of the two procedures are described below. After either procedure, all patients were given an additional 1-day course of oral antibiotics (1 g amoxicillin- 
clavulanate and $500 \mathrm{mg}$ ciprofloxacin).

When undergoing TRUSPB, patients assumed the left lateral position, as shown in Figure 1. Prostate size was measured using a transrectal biplanar ultrasound probe. Subsequently, 10 core biopsies were taken: five cores were taken from each side of the prostate at the base, mid, apex, upper lateral, and lower lateral regions. Each procedure was 5 to 10 minutes in duration.

When undergoing TPUSPB, patients assumed the Lloyd-Davies position prior to injection of lidocaine for LA (described above). After lidocaine injection, a biplanar ultrasound probe was inserted through the anus. The prostate size was measured, and 14-gauge angiocatheters were then inserted at the sites previously used for LA injection, as shown in Figure 2. Ten core biopsies were obtained in a manner similar to that of TRUSPB. Because of the different orientation of the biopsy needle, the apical biopsy was targeted towards the anterior fibromuscular layer. The biopsy needle was maintained parallel to the probe to ensure clear visualisation of the targeted area, which was possible when the whole needle was completely visualised on ultrasound (Fig 3). Each procedure was 10 to 15 minutes in duration. We also assessed the pain experienced during the TPUSPB at three time points, namely during probe insertion into the anus, LA injection, and biopsy procedures, by verbal analogue scale (0-10) during TPUSPB.

Statistical analyses were performed using SPSS (Windows version 24.0; IBM Corp, Chicago [IL], United States). For continuous variables, age was compared by independent $t$ test, while PSA, prostate size, and PSA density were compared by the Mann-Whitney $U$ test as they did not exhibit normal distributions. Normality was assessed by normal QQ

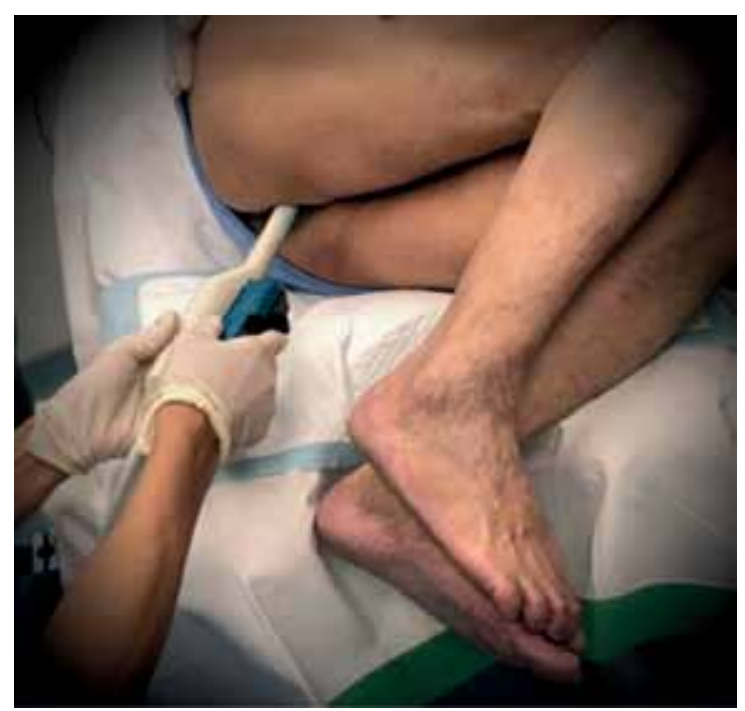

FIG I. Positioning and route for transrectal ultrasoundguided biopsy

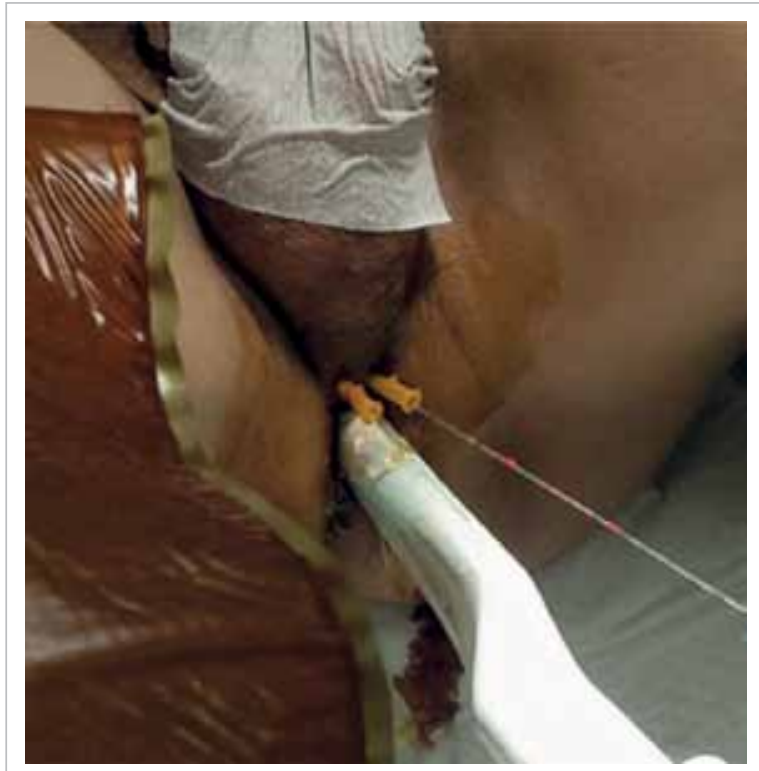

FIG 2. Puncture guides were inserted at sites of local anaesthesia injection

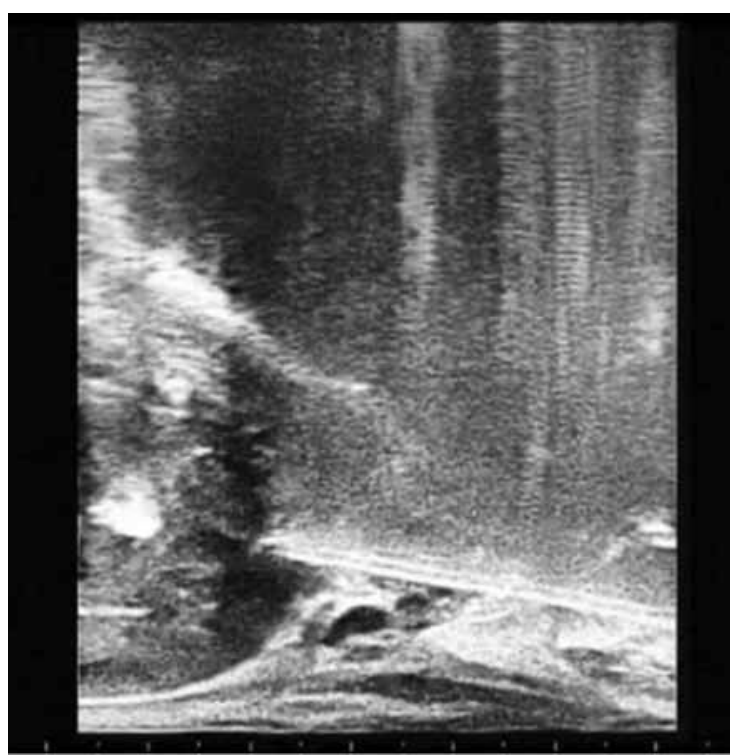

FIG 3. Whole biopsy needle is completely visualised on ultrasound

plots and the Shapiro-Wilk test. When comparing categorical variables, including cancer detection rates and complication rates, the Chi squared test was used if the expected count in each cell was $>5$; otherwise, Fisher's exact test was used. In addition, the Cochran-Mantel-Haenszel test was performed to assess whether there was an association between the biopsy method and cancer detection rate according to clinical stage. Differences with a two-sided $\mathrm{P}$ value of $<0.05$ were considered to be statistically significant. 


\section{Results}

The patient characteristics, prostate cancer detection rates, and complications in patients who underwent TPUSPB, compared with those who underwent TRUSPB, are summarised in Table 1. Age did not significantly differ between the two groups. The median serum PSA in the TPUSPB group was higher than that in the TRUSPB group $(12.0 \mathrm{ng} / \mathrm{dL}$ vs 9.5 $\mathrm{ng} / \mathrm{dL}, \mathrm{P}=0.047$ ). Moreover, the median prostate size in the TPUSPB was smaller than that in the TRUSPB group ( $46.2 \mathrm{~mL}$ vs $56.8 \mathrm{~mL}, \mathrm{P}=0.003$ ). Therefore, the PSA density of TPUSPB group was higher than that in the TRUSPB group ( 0.27 vs $0.16, \mathrm{P}=0.001)$.

Stratified prostate cancer detection rates in patients who underwent TPUSPB, compared with those who underwent TRUSPB, are listed in Table 2. There was no statistically significant difference in overall prostate cancer detection rate between the two groups. In subgroup analysis stratified by serum PSA level, the TPUSPB group had a higher prostate cancer detection rate than the TRUSPB group among patients with 20 to $100 \mathrm{ng} / \mathrm{mL}$ PSA ( $50 \%$ vs $15 \%, \mathrm{P}=0.036$ ). However, there were no statistically significant differences in prostate cancer detection rates among other subgroups according to PSA levels. There were also no statistically significant differences in prostate cancer detection rates between the two groups upon stratification according to PSA density or clinical staging.
In analysis of 35 patients with positive cores in the TPUSPB group, 16 (45.7\%) patients had at least one positive core in the anterior fibromuscular stroma. Among these 16 patients, 14 were diagnosed with high-risk prostate cancers, with multiple positive cores in each patient. The relatively high proportion of high-risk prostate cancer in the TPUSPB cohort might explain the relatively high number of positive cores in the anterior fibromuscular stroma.

In the TPUSPB group, 19 patients had previous negative findings in TRUSPB; three of these 19 (15.7\%) were diagnosed with prostate cancer based on the findings of TPUSPB. Two of the three tumours were detected in the anterior fibromuscular layer, and the remaining tumour was found in the apical zone. In the TRUSPB group, 36 patients had previous negative findings in TRUSPB; six (16.7\%) of these were diagnosed with prostate cancer based on the findings of the current TRUSPB.

Concerning about the pain experienced during TPUSPB, the pain scores reported by patients during probe insertion into the anus, LA injection, and biopsy procedures were $1-2,1-2$, and $2-4$, respectively.

With respect to complications, we initially planned to use the Sepsis-3 described above, but no patients in this study developed clinical signs of infection that met the criteria for sepsis. However, four (4\%) patients in the TRUSPB group developed fever requiring hospital admission compared with

TABLE I. Patient characteristics, prostate cancer detection rates, and complications in patients who underwent TPUSPB compared with those who underwent TRUSPB*

\begin{tabular}{|c|c|c|c|}
\hline & TRUSPB & TPUSPB & $\mathbf{P}$ value \\
\hline Age & $69.1 \pm 8.2$ & $67.7 \pm 6.2$ & 0.154 \\
\hline PSA (ng/dL) & $9.5(6.0-17.0)$ & $12.0(7.6-25.7)$ & 0.047 \\
\hline Prostate size (mL) & 56.8 (39.7-80.7) & $46.2(34.0-58.0)$ & 0.003 \\
\hline PSA density & $0.16(0.11-0.30)$ & $0.27(0.15-0.64)$ & 0.001 \\
\hline \multicolumn{4}{|l|}{ Indications of prostate biopsies } \\
\hline Patients undergoing active surveillance & 4 & 2 & - \\
\hline Elevated serum PSA & 63 & 71 & - \\
\hline Clinically T2 disease & 25 & 22 & - \\
\hline Clinically T3 disease & 8 & 5 & - \\
\hline \multicolumn{4}{|l|}{ Complications required hospitalisation } \\
\hline Fever & $4(4 \%)$ & 0 & 0.121 \\
\hline Retention of urine & $1(1 \%)$ & $3(3 \%)$ & 0.621 \\
\hline Rectal bleeding & $1(1 \%)$ & 0 & 1.000 \\
\hline Haematuria & 0 & 0 & - \\
\hline Prostate cancer detection & $25(25 \%)$ & $35(35 \%)$ & 0.123 \\
\hline
\end{tabular}

Abbreviations: PSA = prostate-specific antigen;TPUSPB = transperineal ultrasound-guided prostate biopsy; TRUSPB = transrectal ultrasound-guided prostate biopsy

* Data are shown as mean \pm standard deviation, median (interquartile range), or No. (\%), unless otherwise specified 
TABLE 2. Stratified prostate cancer detection rates in patients who underwent TPUSPB compared with those who underwent TRUSPB*

\begin{tabular}{lccc}
\hline & TPUSPB (n=35) & TRUSPB (n=25) & P value \\
\hline Overall & $35(35 \%)$ & $25(25 \%)$ & 0.123 \\
\hline Stratified according to PSA level $(\mathrm{ng} / \mathrm{mL})$ & & & 0.147 \\
\hline $4-10$ & $10 / 38(26 \%)$ & $7 / 50(14 \%)$ & 0.819 \\
$>10-20$ & $8 / 32(25 \%)$ & $8 / 29(28 \%)$ & 0.036 \\
$>20-100$ & $13 / 26(50 \%)$ & $2 / 13(15 \%)$ & 1.000 \\
$>100$ & $4 / 4(100 \%)$ & $8 / 8(100 \%)$ & 0.787 \\
\hline Stratified according to PSA density & & & 0.492 \\
\hline$<0.1$ & $1 / 13(8 \%)$ & $2 / 19(11 \%)$ & 0.863 \\
\hline$>0.1-0.2$ & $4 / 23(17 \%)$ & $5 / 44(11 \%)$ & 1.000 \\
$>0.2$ & $30 / 64(47 \%)$ & $18 / 37(49 \%)$ & 0.600 \\
\hline Stratified according to clinical staging & & & 0.210 \\
\hline Patient under active surveillance with T1 disease & $1 / 2(50 \%)$ & $1 / 4(25 \%)$ & 1.000 \\
\hline Normal digital rectal examination & $8 / 71(11 \%)$ & $9 / 63(14 \%)$ & $17 / 25(68 \%)$ \\
\hline Clinically T2 disease & $11 / 22(50 \%)$ & $8 / 8(100 \%)$ & \\
\hline Clinically T3 disease & $5 / 5(100 \%)$ & & \\
\hline
\end{tabular}

Abbreviations: PSA = prostate-specific antigen;TPUSPB = transperineal ultrasound-guided prostate biopsy;TRUSPB = transrectal ultrasound-guided prostate biopsy

* Data are shown as No. (\%), unless otherwise specified

TABLE 3. Clinical findings of four patients in the TRUSPB group who had fever requiring admission

\begin{tabular}{lcccccccc}
\hline & $\begin{array}{c}\text { Fever with highest } \\
\text { temperature }\left({ }^{\circ} \mathbf{C}\right)\end{array}$ & $\begin{array}{c}\text { Respiratory rate } \\
\text { per minute }\end{array}$ & BP & $\begin{array}{c}\text { Pulse rate } \\
\text { per } \mathbf{m i n}\end{array}$ & GCS & $\begin{array}{c}\text { WCC } \\
(\times \mathbf{1 0} / \mathbf{L})\end{array}$ & $\begin{array}{c}\text { Blood or urine } \\
\text { culture }\end{array}$ & $\begin{array}{c}\text { Admission } \\
\text { duration (days) }\end{array}$ \\
\hline Case 1 & 39.1 & 16 & $120 / 80$ & 99 & 15 & 21.8 & No growth & 8 \\
Case 2 & 39.6 & 18 & $98 / 52$ & 109 & 15 & 17.3 & No growth & 9 \\
Case 3 & 39.5 & 17 & $130 / 72$ & 110 & 15 & 15.6 & No growth & 7 \\
Case 4 & 38.6 & 15 & $125 / 92$ & 102 & 15 & 22.5 & ESBL E coli & 8 \\
\hline
\end{tabular}

Abbreviations: BP = blood pressure; ESBL E coli = extended-spectrum beta-lactamase Escherichia coli; GCS = Glasgow Coma Scale; TRUSPB = transrectal ultrasound-guided prostate biopsy; WCC = white cell count

none in the TPUSPB group ( $\mathrm{P}=0.121)$ [Table 3]. At thus preventing infections associated with least 1 week of intravenous antibiotic treatment was TRUSPB; however, fluoroquinolone-resistant bacteria prescribed for all four of those patients with fever. and extended-spectrum beta-lactamase-producing Three patients in the TPUSPB group and one in the bacteria are present within the intestinal flora of TRUSPB group developed acute retention of urine $40.4 \%$ and $41.0 \%$ of Chinese patients, respectively. ${ }^{13}$ $(\mathrm{P}=0.621)$. No patients in the TPUSPB group and one Accordingly, the rate of post-TRUSPB sepsis is in the TRUSPB group had rectal bleeding $(\mathrm{P}=1.000)$. rising both in Hong Kong ${ }^{6}$ and worldwide. ${ }^{14}$ To There were no admissions due to haematuria in reduce the rates of infection, many strategies have either group.

\section{Discussion}

Since Hodge et $\mathrm{al}^{2}$ introduced the systematic sextant biopsy protocol, TRUSPB has become the main approach to detect prostate cancer worldwide. In recent years, many urologists have described increased risks of infection and sepsis associated with TRUSPB. ${ }^{12}$ Fluoroquinolone was previously been attempted, including augmented prophylactic antibiotic protocols. However, no strategies have prevented the development of sepsis due to the transfer of faecal bacteria into the blood stream through TRUSPB puncture sites. ${ }^{10}$ Transperineal ultrasound-guided prostate biopsy has been suggested as a potentially safer alternative. Notably, the indications, workups, medications, and numbers of cores are identical between TRUSPB and TPUSPB. thought to provide effective antibiotic prophylaxis, However, the techniques differ with respect to 
multiple aspects, including patients' position and puncture route, ${ }^{15}$ as described in the Methods section of this paper.

From the pain scores recorded during TPUSPB, most patients tolerated the procedure well. Because the entire procedure was performed under LA, all patients could be discharged on the same day without the need for general anaesthesia or monitored anaesthesia care. In Asian nations, many patients must travel a considerable distance to reach the hospital; therefore, 1-day out-patient procedures are preferable for patients and their relatives. Moreover, some patients are high-risk or unfit for general anaesthesia or monitored anaesthesia care; procedures performed under LA are therefore much safer and more practical for them.

Transrectal ultrasound-guided prostate biopsy neglects prostate cancer located in the anterior fibromuscular stroma, whereas the TPUSPB does not. ${ }^{16}$ In our study, a significant proportion of positive prostatic cores were found in the anterior fibromuscular stroma among patients in the TPUSPB group. Moreover, some patients with prior negative findings in TRUSPB were diagnosed with prostate cancer in the anterior fibromuscular stroma based on the results of TPUSPB. Therefore, TPUSPB may have an advantage over TRUSPB in patients with previous negative biopsy findings.

This study had some limitations. First, a consistent number of cores was biopsied in all patients in the TPUSPB group, irrespective of prostate size. To improve the rate of prostate cancer detection, some experts have advocated for the use of different numbers of prostate biopsies, based on prostate size ${ }^{16}$-more biopsies should be taken in patients with larger prostates. Second, TPUSPB required more time than TRUSPB. However, as each step is standardised, the duration of the procedure may decrease. Third, there was no documentation of pain scores in the TRUSPB group; thus, a comparison could not be performed. Future studies should address these limitations. In particular, a larger sample size is needed to confirm whether TPUSPB is superior with respect to the rate of prostate cancer detection.

\section{Conclusion}

In summary, TPUSPB avoids penetration of the rectal mucosa and possible transfer of intestinal flora to the blood stream during the procedure. This contributed to the lack of infections in the present study. With respect to prostate cancer detection rate, TPUSPB is at least comparable to TRUSPB. Therefore, an increasing number of urologists may adopt this technique in the future.

\section{Author contributions}

All authors had full access to the data, contributed to the study, approved the final version for publication, and take responsibility for its accuracy and integrity.

Concept or design of study: KL Lo, KL Chui, CF Ng. Acquisition of data: KL Lo, K Lim, JKM Li, J Wong, SK Mak. Analysis or interpretation of data: KL Lo, CF Ng, SCH Leung, SF Ma.

Drafting of the manuscript: KL Lo, CF Ng.

Critical revision for important intellectual content: All authors.

\section{Conflicts of interest}

As an editor of the journal, CF Ng was not involved in the peer review process. Other authors have disclosed no conflicts of interest.

\section{Acknowledgement}

We would like to thank for the support of the nursing staff in the Integrated Ambulatory Care Centre, North District Hospital for their support to the procedures.

\section{Funding/support}

This research received no specific grant from any funding agency in the public, commercial, or not-for-profit sectors.

\section{Ethics approval}

This study was approved by the Joint Chinese University of Hong Kong-New Territories East Cluster Clinical Research Ethics Committee (Ref CREC 2018.323).

\section{References}

1. Hong Kong Cancer Registry, Hospital Authority, Hong Kong SAR Government. Prostate cancer in 2016. Available from: http://www3.ha.org.hk/cancereg/pdf/top10/rank_ 2016.pdf. Accessed 17 May 2019.

2. Hodge KK, McNeal JE, Terris MK, Stamey TA. Random systematic versus directed ultrasound guided transrectal core biopsies of the prostate. J Urol 1989;142:71-4.

3. Heidenreich A, Bastian PJ, Bellmunt J, et al. EAU guidelines on prostate cancer. Part 1: screening, diagnosis, and local treatment with curative intent-update 2013. Eur Urol 2014;65:124-37.

4. Toner L, Bolton DM, Lawrentschuk N. Prevention of sepsis prior to prostate biopsy. Investig Clin Urol 2016;57:94-9.

5. Chan ES, Lo KL, Ng CF, Hou SM, Yip SK. Randomized controlled trial of antibiotic prophylaxis regimens for transrectal ultrasound-guided prostate biopsy. Chin Med J (Engl) 2012;125:2432-5.

6. Ng CF, Chan SY. Re: the incidence of fluoroquinolone resistant infections after prostate biopsy-are fluoroquinolones still effective prophylaxis? J Urol 2008;180:1570-1.

7. Kojima M, Hayakawa T, Saito T, Mitsuya H, Hayase Y. Transperineal 12-core systematic biopsy in the detection of prostate cancer. Int J Urol 2001;8:301-7.

8. Xue J, Qin Z, Cai H, et al. Comparison between transrectal and transperineal prostate biopsy for detection of prostate cancer: a meta-analysis and trial sequential analysis. Oncotarget 2017;8:23322-36.

9. Chang DT, Challacombe B, Lawrentschuk N. Transperineal biopsy of the prostate-is this the future? Nat Rev Urol 2013;10:690-702. 
10. Grummet JP, Weerakoon M, Huang S, et al. Sepsis and 'superbugs': should we favour the transperineal over the transrectal approach for prostate biopsy? BJU Int 2014;114:384-8.

11. Singer M, Deutschman CS, Seymour CW, et al. The Third International Consensus Definitions for Sepsis and Septic Shock (Sepsis-3). JAMA 2016;315:801-10.

12. Steensels D, Slabbaert K, De Wever L, Vermeersch P, Van Poppel $\mathrm{H}$, Verhaegen J. Fluoroquinolone-resistant $E$. coli in intestinal flora of patients undergoing transrectal ultrasound-guided prostate biopsy-should we reassess our practices for antibiotic prophylaxis? Clin Microbiol Infect 2012;18:575-81.

13. Tsu JH, Ma WK, Chan WK, et al. Prevalence and predictive factors of harboring fluoroquinolone-resistant and extended-spectrum $\beta$-lactamase-producing rectal flora in Hong Kong Chinese men undergoing transrectal ultrasound-guided prostate biopsy. Urology 2015;85:15-21.

14. Williamson DA, Barrett LK, Rogers BA, Freeman JT, Hadway P, Paterson DL. Infectious complications following transrectal ultrasound-guided prostate biopsy: new challenges in the era of multidrug-resistant Escherichia coli. Clin Infect Dis. 2013;57:267-74.

15. Emiliozzi P, Corsetti A, Tassi B, Federico G, Martini M, Pansadoro V. Best approach for prostate cancer detection: a prospective study on transperineal versus transrectal sixcore prostate biopsy. Urology 2003;61:961-6.

16. Ong WL, Weerakoon M, Huang S, et al. Transperineal biopsy prostate cancer detection in first biopsy and repeat biopsy after negative transrectal ultrasound-guided biopsy: the Victorian Transperineal Biopsy Collaboration experience. BJU Int 2015;116:568-76. 